\title{
Contour Completion of Partly Occluded Objects Based on Figural Goodness
}

\author{
Takahiro Hayashi \\ Institute of Science and Technology, Niigata University, Japan \\ hayashi@ie.niigata-u.ac.jp \\ Tatsuya Ooi \\ Graduate School of Science and Technology, \\ Niigata University, Japan \\ f15c007g@mail.cc.niigata-u.ac.jp \\ Motoki Sasaki \\ Graduate School of Science and Technology, \\ Niigata University, Japan \\ f14c019f@mail.cc.niigata-u.ac.jp
}

\begin{abstract}
Object extraction has a crucial role in various visual semantic scenarios. In this paper, we propose a system for object extraction which can deal with an object partly occluded by other objects. In order to estimate the hidden part of the occluded object, the system combines different types of contour completion methods such as curve completion and symmetry completion. The system is composed of two modules: contour discrimination and contour completion. The contour discrimination module separates the contour of the occluded object from the contours of the occluding objects. To the contour of the occluded object, the contour completion module applies different types of contour completion algorithms to generate various completion patterns. Based on the perceptual model of figural goodness, the system computes the figural goodness scores of the generated patterns, where the simplicity and symmetricity are evaluated. Finally, the system outputs the pattern having the highest score as a result of the object extraction. From the experimental results, we have confirmed that the figural goodness model works effectively for extracting partly occluded objects.
\end{abstract}

Keywords: Object decomposition, contour completion, figural goodness

\section{Introduction}

Object extraction has a crucial role in various contemporary visual semantics scenarios (including scene analysis, object recognition, image retrieval, raster-to-vector image conversion, etc.) [1-7]. Object extraction is implemented in various software products of image processing such as Live Trace in Adobe Illustrator and PowerTrace in CorelDRAW.
Conventional object extraction methods are based on the technique of line tracing. However, there is a limitation that they cannot properly interpret the contour of an object which is partly occluded by other objects.

When humans look at an object which is partly occluded by other objects, they can correctly estimate the hidden part of the contour of the occluded object. This human visual perception is known as amodal completion in Gestalt psychology [8-11]. While humans estimate the hidden part of a contour, computers just 


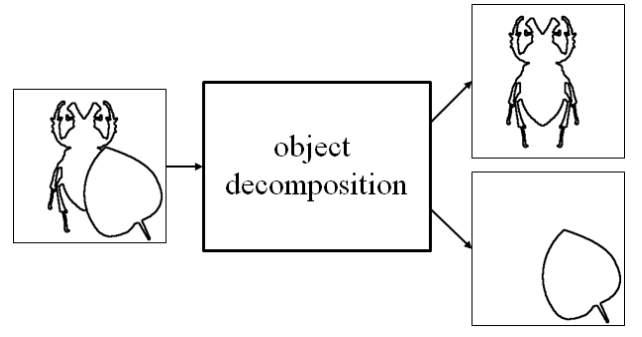

Fig. 1. object decomposition considering visual occlusion

trace the visible part of contours to decompose objects, which limits the ability of object extraction by computers.

In order to extract partly occluded objects correctly, it is necessary to model the phenomenon of amodal completion. The goals of our study are to develop and evaluate an object decomposition system modeling human visual completion as shown in Fig. 1.

In the fields of computer vision and image processing, there are some pilot studies which tackle the challenge of computationally modeling amodal completion [12-20]. However, the application fields of these computational methods are severely limited because whether these methods work effectively or not depends on the shape class of an object. For example, completion methods proposed in $[12,13]$ are based on the technique of interpolation with a curve such as a Bspline curve. These methods work effectively to an object which has a smooth contour (i.e., there are no nondifferentiable points on the contour). On the other hand, these methods cannot properly deal with objects having more complex contours like the input image in Fig. 1.

In [18-20], completion methods considering symmetry structures of an object are proposed. However, these methods work effectively when objects have symmetry structures.

Since the effectiveness of conventional completion methods are sensitively affected by the shape class of an input object, in this paper, we combine different types of completion methods for processing objects independently from their shape classes.

There are two possible approaches for combining completion methods. One is a preprocessing approach and the other is a post-processing approach.

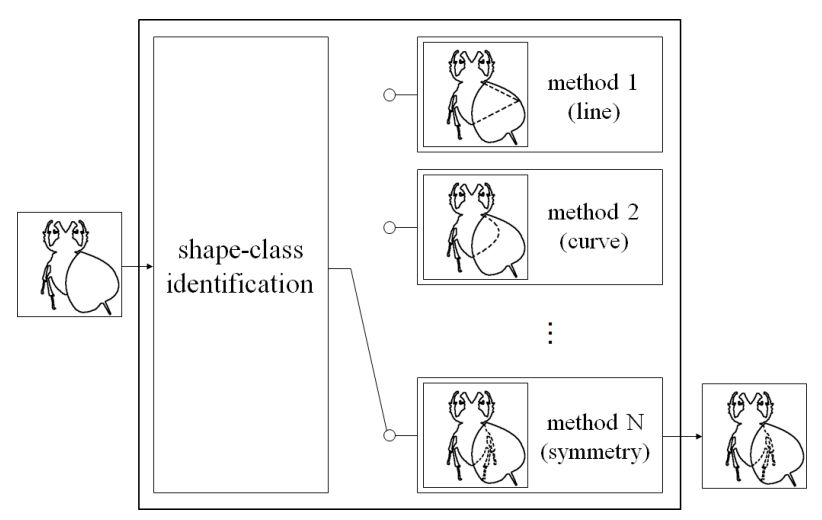

Fig. 2. Preprocessing approach for object decomposition in which completion methods are switched based on the shape class of the input image

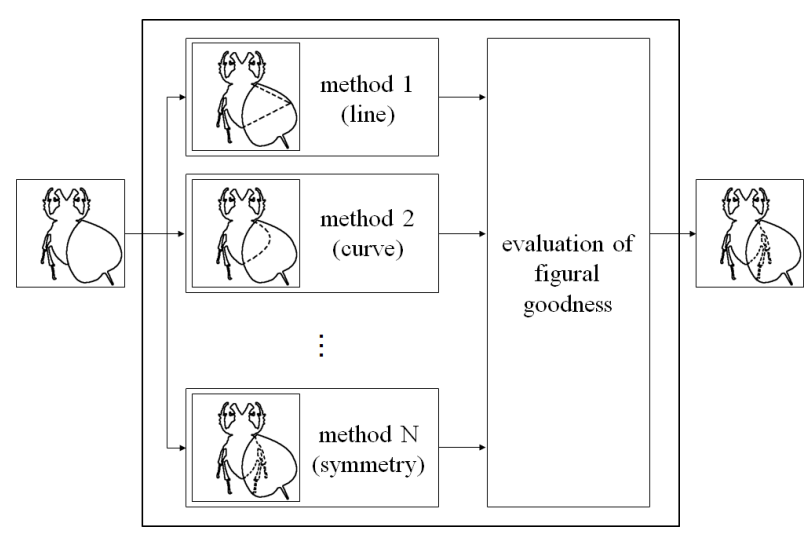

Fig. 3. Post-processing approach for object decomposition in which candidate completion patters are generated by applying all the completion methods, then the best completion pattern is selected based on figural goodness

As shown in Fig. 2, in the preprocessing approach, the shape class of an input image is identified before a completion method is executed. Based on the identification result an appropriate completion method is selected to execute. The difficulty here is that shape class identification is not always easy for computers because many factors influences the results of identification and many exceptions are possibly contained in each shape class.

In the post-processing approach, all the completion methods are executed to generate various completion patterns as candidates. From them the best one is selected from the aspect of figural goodness. Fig. 3 shows the outline of the post-processing approach. 
Figural goodness of each completion pattern is evaluated based on a model of the human perception on good figures. According to the law of Prägnanz in Gestalt psychology, objects are seen in a way that makes them appear as simple as possible [8]. Focusing on the characteristics of human perception, in this approach the simplicity and symmetricity of each completion pattern are evaluated. The post-processing approach has a merit that once a scoring model of figural goodness is defined, an object decomposition system can be developed easily. In addition, this approach is independent from individual completion methods. Due to the merits, this paper adopts the postprocessing approach for constructing an object decomposition system.

The contributions of this paper are as follows.

(i) The proposed system can expand application fields of object decomposition because the restrictions on shape classes can be removed.

(ii) The proposed system improves the usefulness of existing image retrieval systems, pattern recognition systems, drawing software products because object decomposition function becomes more intelligent and practical.

The rest of the paper is organized as follows. In Section 2, we describe related works. In Section 3, we propose an object decomposition system which combines different types of completion methods and employs a scoring method for evaluating figural goodness. In section 4, we show the results of experiments we conducted for confirming the effectiveness and limitations of the system. Finally, we conclude the paper and describe future works in Section 5 .

\section{Related Works}

The phenomenon of amodal completion has long been studied in the field of Gestalt psychology [8-11]. These studies have revealed that local and global structures of a shape influence generated completion patterns.

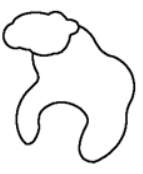

(a)

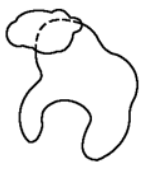

(b)

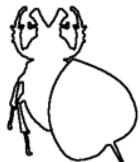

(c)

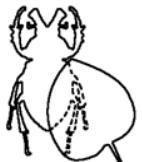

(d)
Fig. 4. Completion considering local shape structures and global shape structures
For example, when humans see the image shown in Fig. 4(a), they can estimate a simple curve as a completion pattern like Fig. 4(b). In the completion process, humans focus on local structures of the shape such as the continuity and smoothness of the contour in a local region. Fig. 4(c) and (d) shows an example of completion patterns generated by considering global shape structures. When humans see the image shown in Fig. 4(c), they can accurately estimate the hidden part like Fig. 4(d). In the completion process, humans consider the line symmetry structure, i.e., a global structure of the shape.

Some computational methods for amodal completion have been proposed. These methods can be classified into two approaches considering local shape structures [12-17] and considering global shape structures [18-20].

Curve completion and line completion are two major completion methods considering local shape structures [12-14]. In the curve completion approach, the problem of contour completion is regarded as a problem of interpolation. The gap between the endpoints of the occluded contour is interpolated with a parametric curve such as a B-spline curve. In the line completion approach, two lines are extended from the endpoints of the contour toward the direction of the tangent line at the endpoints.

Line symmetry completion method [18, 19] and rotational symmetry completion [20] are methods for contour completion considering global shape structures. In these methods, symmetry parameters (i.e., linesymmetry axes, the angle of rotation, the center of rotation, etc.) are estimated from the visible part of the contours of an occluded object. Based on the symmetry parameters, the symmetry points of the hidden part are detected. Using the symmetry points as a patch, the hidden part is reconstructed.

These contour completion methods have a problem that shape classes of input images are restricted, which limits applications of object decomposition. In our study, different types of completion methods are combined to develop an object decomposition system which can deal with any shapes.

In our system, completion patterns generated by using multiple completion methods are evaluated from the aspect of figural goodness. The factors of figural goodness have been studied in the field of Gestalt 
psychology and cognitive science on human visual perception. These studies showed that simplicity and symmetricity are main factors of good figures [21]. In [22], Shimaya proposed a qualitative method for evaluating the figural goodness of a completion pattern from the aspects of simplicity and symmetricity. We improve and computationally model the Shimaya's method to actualize the scoring part of completion patterns.

\section{Proposed System}

\subsection{Outline}

The input of the proposed system is an image in which an object is partly occluded by other objects. The output of the system is a decomposition pattern of the image. The proposed system executes the following five steps in turn:

(step 1) Receive an image in which an object is partly occluded by other objects.

(step 2) Discriminate the contours of occluding and occluded objects from the input image as shown in Fig. 5.The details of this step are explained in Sec 3.2.

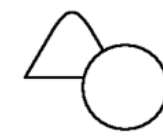

input image
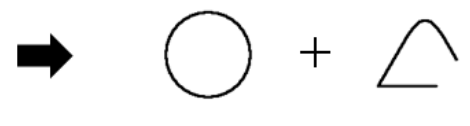

occluding object

occluded object
Fig. 5. Discrimination of occluding and occluded objects.

(step 3) Generate candidate completion patterns of the occluded object using the following four completion methods.

method 1: line completion [14]

method 2: curve completion (B-spline interpolation)

method 3: line symmetry completion [19]

method 4: rotational symmetry completion [20]

(step 4) Evaluate the figural goodness score of each completion pattern. The details of this step are explained in Sec 3.3.

(step 5) Output the completion pattern gained the highest figural goodness score as the best completion pattern.

\subsection{Contour discrimination}

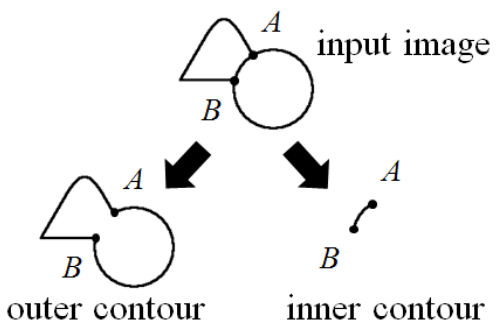

Fig. 6. Extraction of outer and inner contours

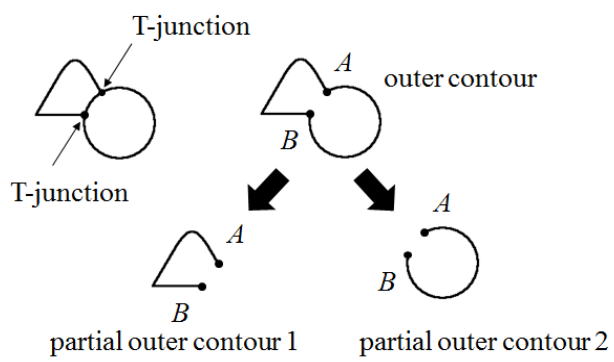

Fig. 7. Division of outer contour
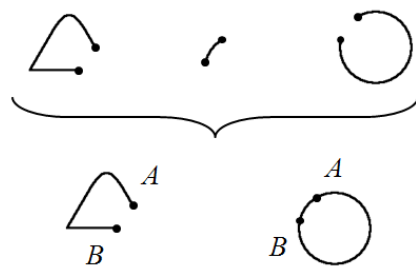

occluded object occluding object

Fig. 8. Identification of occluding and occluded objects

This subsection explains the method for discriminating the contour of the occluded object from the one of the occluding object in an image. The following five steps are processed in turn to discriminate the contours.

(step 1) (Extraction of outer and inner contours) Extract the outmost contour from the input image with the boundary tracing. The outmost contour is called the outer contour and the other part of contour is called the inner contours. Fig. 6 shows an example of outer and inner contours. 
(step 2) (Division of outer contour) Divide the outer contour extracted in step linto partial outer contours at the points of $\mathrm{T}$-junctions and $\mathrm{X}$-junctions of the input image. Fig. 7 shows an example of the process of division of outer contour.

(step 3) (Identification of occluding and occluded objects) In the partial outer contours obtained in step 2, detect the one which can be smoothly connected with the inner contour, and then actually connect the detected partial contour with the inner contour to obtain the contour of the occluding object. The other partial outer contour can be identified as the contour of the occluded object. Fig. 8 shows the process of identification of occluding and occluded objects.

\subsection{Evaluation of figural goodness}

The figural goodness of each completion pattern is evaluated from the aspect of simplicity and symmetricity. We computationally model Shimaya's quantitative method [22] for scoring figural goodness. According to the model, the figural goodness $g$ of a completion pattern $L$ is defined as

$g(L)=1-\frac{n_{c}(L)+1}{n_{c}(L)+2} \cdot \min \left\{\frac{1}{n_{a}(L)+1}, \frac{1}{n_{o}(L)}\right\}$

where $n_{\mathrm{c}}(L)$ is the number of corners, $n_{\mathrm{a}}(L)$ is the number of symmetry axes and $n_{\mathrm{o}}(L)$ is the order of rotational symmetry. The order of rotational symmetry is defined as $360^{\circ} / \theta$, where $\theta$ is the angle of rotation.

\begin{tabular}{|c|c|c|c|c|}
\hline$L$ & & & & \\
\hline$n_{c}(L)$ & 0 & 3 & 23 & 47 \\
\hline$n_{a}(L)$ & $\infty$ & 3 & 1 & 0 \\
\hline$n_{o}(L)$ & $\infty$ & 3 & 1 & 1 \\
\hline$g(L)$ & 1 & 0.8 & 0.52 & 0.02 \\
\hline
\end{tabular}

Fig. 9. Figural goodness of some figures

In the scoring model, the figural goodness of a shape is evaluated from the aspect of the simplicity and symmetricity of a shape. The simplicity is evaluated from the number of corner $n_{\mathrm{c}}(L)$ and the symmetricity is evaluated from symmetry parameters $n_{\mathrm{a}}(L)$ and $n_{\mathrm{o}}(L)$.
When a perfect circle $\left(n_{\mathrm{c}}(L)=0, n_{\mathrm{a}}(L)=\infty, n_{\mathrm{o}}(L)=\infty\right.$ is input, the score reaches the maximum. Fig. 9 shows the scores of figural goodness of some images. From the figure, we confirm that simpler images have higher scores.

In order to compute $n_{\mathrm{c}}(L), n_{\mathrm{a}}(L)$ and $n_{\mathrm{o}}(L)$, a corner detection method [23], a line symmetry detection method [24] and a rotational symmetry detection method [25, 26] are used, respectively.

We have confirmed that the scoring by Eq. (1) works effectively in most cases. However, from a preliminary experiment, we have found there are some cases when the scoring results does not match the human feeling on figural goodness.

\begin{tabular}{|c|c|c|}
\hline & (a) & (b) \\
\hline$L$ & \\
\hline$\theta$ & $90^{\circ}$ & $30^{\circ}$ \\
\hline$g(L)$ & 0.550 & 0.556 \\
\hline$g^{\prime}(L)$ & 0.700 & 0.556 \\
\hline
\end{tabular}

Fig. 10. Influence of the angle of a symmetry axis to figural goodness

Fig. 10 shows an example. The figure shows two possible completion patterns (a) and (b). Pattern (a)is generated by assuming a vertical symmetry axis and pattern (b) is generated by taking a symmetry axis along with the direction of $30^{\circ}$. In the two completion patterns, pattern (a) is more frequently generated by many people than pattern (b). This result indicates that the existence of the vertical symmetry axis increases the figural goodness of the shape. Since the score defined by Eq. (1) does not take the influence of the angle of a symmetry axis into account, the figural goodness $g(L)$ of pattern (a) is lower than pattern (b).

To consider the influence of the vertical symmetry axis, we improve the scoring of figural goodness as follows:

$g^{\prime}(L)=1-\frac{n_{c}(L)+1}{n_{c}(L)+2} \cdot \min \left\{\frac{1}{n_{a}(L)+m_{a}(L)+1}, \frac{1}{n_{o}(L)}\right\}$ 


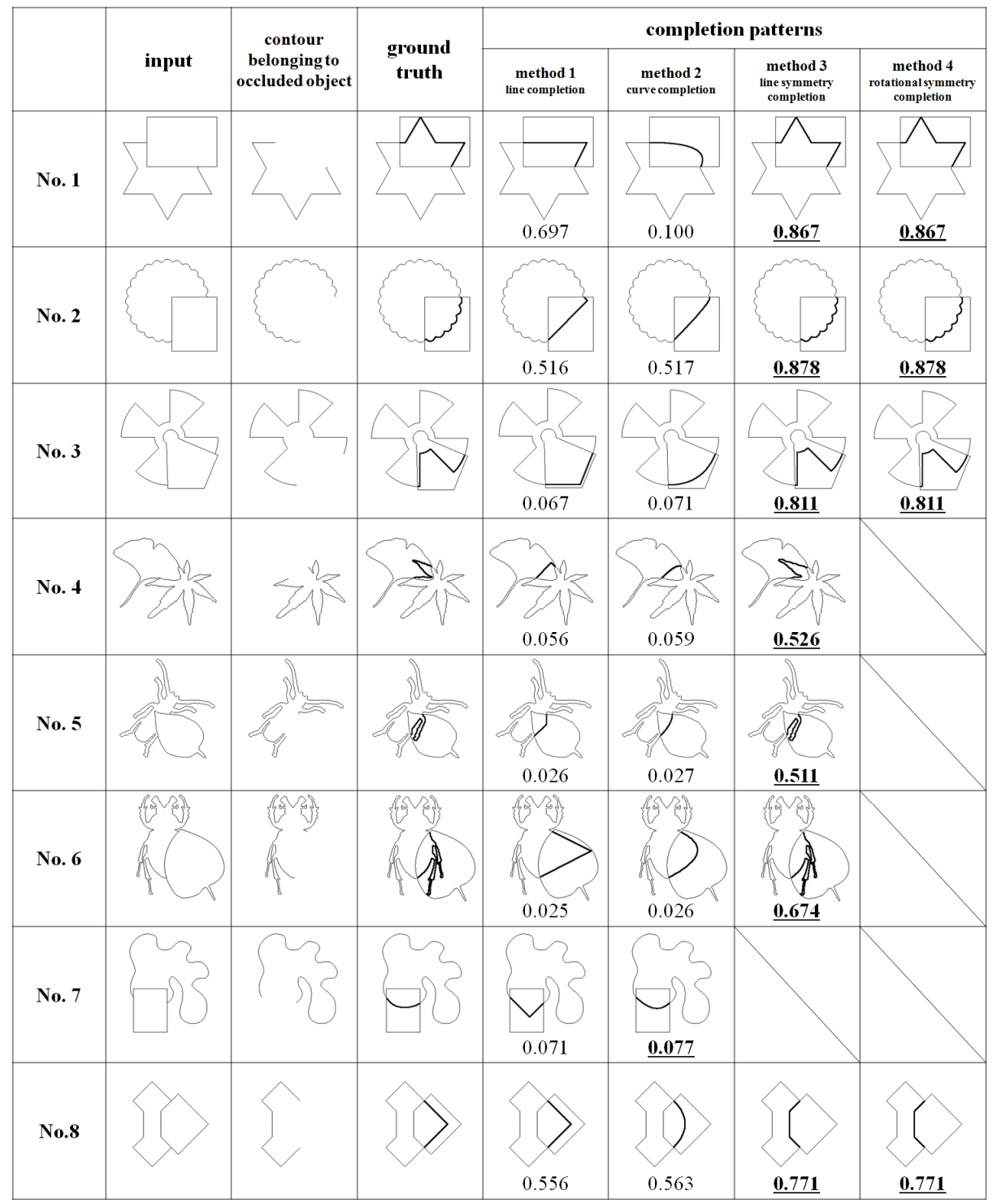

Fig. 11. Experimental results: figural goodness scores are shown below each completion pattern and the best ones (the output of the system) are underlined.

where $m_{\mathrm{a}}(L)$ is the additional parameter evaluating the influence of the vertical symmetry axis. $m_{\mathrm{a}}(L)$ is defined as follows:

$$
m_{a}(L)= \begin{cases}1 & \text { if } \exists a \in A(L), \theta(a)=90^{\circ} \\ 0 & \text { otherwise }\end{cases}
$$

where $A(L)$ is the set of the line symmetry axes of $L$, $\theta(a)$ is the angle of line symmetry axis $a . m_{\mathrm{a}}(L)$ takes the value 1 only when the shape has the vertical symmetry axis. Otherwise, it has no effect to the figural goodness score.

As shown in Fig. 10, the improved figural goodness $g^{\prime}(L)$ of pattern (a) is higher than pattern (b), which means that the improved scoring better matches human perception on figural goodness.

\section{Experimental Results}

We experimentally evaluated the effectiveness of the proposed system. In the experiment, we compared the 
object decomposition results of the system with the ground truth (i.e., the correct object decomposition generated manually).

Fig. 11 shows the input images used in the experiment, the contours of the occluded objects discriminated from the input images, the ground truth patterns, and four completion patterns generated by each completion method. The numbers shown under each completion pattern represent the figural goodness scores of the pattern. The best scores are underlined.

As shown in Fig. 11, the decomposition results of No. 1 to No. 5 images match with the ground truth, respectively. From these results, we can confirm that the proposed system can select appropriate completion patterns and the scoring model of figural goodness works effectively in these images.

The occluded objects of No. 1, 2 and 3 images have a line symmetry structure and a rotational symmetry structure. Therefore, both the completion patterns generated by method 3 (line symmetry completion) and method 4 (rotational symmetry completion) have the highest figural goodness scores.

The occluded objects of No. 4, 5 and 6 images have line symmetry structures. Therefore, the completion pattern of method 3 has the best scores.

The occluded object of No. 7 image has a characteristic that its contour is smooth (i.e., there is no nondifferentiable points on the contour). Therefore, method 2 (curve completion) can generate the best completion pattern.

To No. 8 image, the system selected pattern 3 (the completion pattern generated by method 3 ) as output. However, the ground truth matches with pattern 1 (the completion pattern generated by method 1 (line completion)). That is, to No. 8 image, the selection of completion pattern failed. Pattern 1 has 7 corners and its order of rotation is 1 . On the other hand, pattern 3 has 10 corners and its order of rotation is 2 . Focusing on the number of corners, pattern 1 is simpler than pattern 3 . However, focusing on the order of rotation, pattern 3 is better figure. It depends on input shapes which factors have stronger influence to figural goodness. In the proposed scoring (Eq. (2)), the factor of symmetry parameters rather than the number of corners is more emphasized. As a consequence of the property of the scoring, the system was not able to select proper completion pattern to No. 8 image. It is necessary to

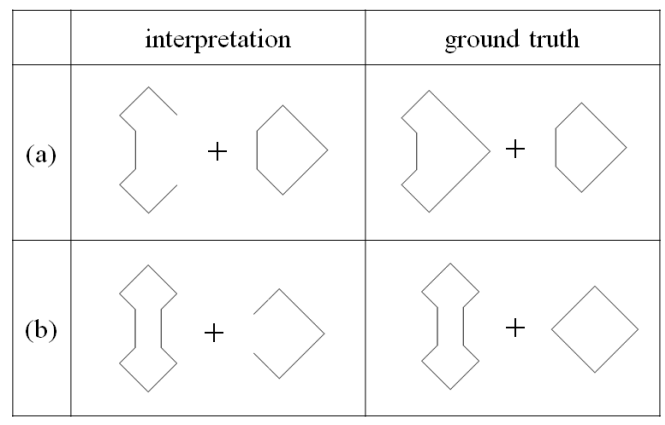

Fig. 12. Two different interpretations of occluding and occluded objects in No. 8 image

improve the score of figural goodness by taking this aspect into account.

In the experiment, we assumed an input image can be uniquely decomposed into occluding and occluded objects. No. 8 image, however, has two different interpretations in object contour discrimination as shown in Fig. 12. Generally, humans can recognize various possible decomposition patterns to one image, and then unconsciously selects one pattern from them as a proper interpretation. However, the selection is not always the same between persons. For taking such personal difference into account, improvement of the proposed system is necessary as a future work.

\section{Conclusions}

In this paper, we have proposed an object decomposition system which can deal with partly occluded objects. In order to develop shape-independent object decomposition, the system combines different types of contour completion methods, namely, line completion, curve completion, line-symmetry completion and rotational symmetry completion. The system evaluates figural goodness of each completion pattern generated by these completion methods from the aspect of simplicity and symmetricity, and then selects the best one.

From the experimental results, we have confirmed that the proposed system can correctly decompose objects from an input image in many cases. However, we have found the scoring model of figural goodness has a limitation that symmetricity tends to be more emphasized than simplicity, which sometimes leads to failures of object decomposition. 
As a future work, we would like to improve the figural goodness scoring by balancing the simplicity and symmetricity depending on an input shape.

Object extraction has a crucial role in various systems of computer vision and image processing. The proposed method contributes to improve the usefulness of these systems because the function of object extraction becomes more intelligent and practical. Besides, the proposed method is independent form the shape classes of input images, which expands application fields. Further studies on pursuing more effective applications of object extraction remains as a future work.

\section{Acknowledgements}

This work was supported by JSPS KAKENHI grant number 26330130.

\section{References}

1. W. Geisler and J. Perry, Contour Statistics in Natural Images: Grouping across occlusions, Visual Neuroscience, 26(2009), pp. 109-121.

2. T. Hayashi, T. Kiyono, K. Abe, and R. Onai, Retrieval of $2 \mathrm{D}$ vector images by matching weighted feature points, in Proc. 15th IEEE Int. Conf. Image Processing, (San Diego, CA, USA, 2008), pp. 961-964.

3. R. Fujita, T. Hayashi, Vector image retrieval based on approximation of Bezier curves with line segments, Proc. IEEE Pacific Rim Conf. Communications, Computers and Signal Processing, (Victoria, Canada, 2011), pp. 431-436.

4. K. Fukushima, Recognition of partly occluded patterns: a neural network model, Biological Cybernetics, 84(4)(2001), pp. 251-259.

5. J. Eakins, J. Boardman, M. Graham, Similarity retrieval of trademark images, IEEE Multimedia, 5(2)(1998), pp. 53-63.

6. Q. Zhang, Qian K. N. Ngan, Segmentation and tracking multiple objects under occlusion from multiview video, IEEE Trans. Image Processing, 20(11)(2011), pp. 33083313.

7. A. Andreopoulosa, J. K. Tsotsosb, 50 Years of object recognition: Directions forward, Computer Vision and Image Understanding, 117(8)(2013), pp. 827-891

8. G. Kanizsa, Organization in Vision: Essays on Gestalt Perception, (Praeger Publishers, Westport, 1979.)

9. F. Boselie, Local and global factors in visual occlusion, Perception, 23(5) (1994), pp. 517-528.

10. A. B. Sekuler, Local and global minima in visual completion: effects of symmetry and orientation, Perception, 23(5) (1994), pp. 529-545.
11. R. van Lier, P. van der Helm, and E. Leeuwenberg, Integrating global and local aspects of visual occlusion, Perception, 23(8) (1994), pp. 883-903.

12. M. Teranishi, N. Ohnishi, and N. Sugie, Subjective contours are useful for extracting contours with very weak contrasts," Proc. 1993 Int. Joint Conf. Neural Network, (1993), pp. 139-142.

13. G. Ben-Yosef and O. Ben-Shahar, Minimum length in the tangent bundle as a model for curve completion, Proc. IEEE Computer Vision and Pattern Recognition, (2010), pp. 2384-2391.

14. D. P. Huttenlocher and S. Ullman, Recognizing solid objects by alignment with an image, Int. J. Computer Vision, 5(2) (1990), pp. 195-212.

15. D. Shoham and S. Ullman, Aligning a model to an image using minimal information," Proc. 2nd Int. Conf. Computer Vision, (1988), pp. 259-263.

16. L. R. Williams and D. W. Jacobs, Euclidean group invariant computation of stochastic completion fields using shiftable-twistable functions, J. Math. Imaging and Vision, 21(2) (2000), pp. 100-116.

17. O. Hirose and T. Nagao, Anisotropic potential field maximization model for subjective contour from line figure, Proc. 3rd Int. Conf. Advances in Visual Computing (Part 1), (2007), pp. 310-320.

18. T. Hayashi, S. Ueki and K. Abe, Contour completion of a partially occluded object in raster-vector conversion, IEICE Trans. Information and Systems, J96-D(11) (2013), pp. 2804-2814.

19. T. Hayashi, M. Sasaki, Contour completion of partially occluded skew-symmetry objects, Proc. IEEE Int. Symp. Multimedia, (Taichung, Taiwan, 2014), pp. 90-93.

20. T. Hayashi and M. Kato, Contour completion of partly occluded objects for more natural raster-vector conversion, Proc. IEEE Conf. Image Processing, (Melbourne, Australia, 2013), pp. 3527-3531.

21. K. T. Spoehr and S. W. Lehmkule, Visual information Processing, (Freeman and Company, New York, 1982).

22. A. Shimaya, Interpreting non-3-D line drawings, Artificial Intelligence, 77(1) (1995), pp. 1-41.

23. M. Sarfraz, Z. N. K. Swati, S. Zaman, A novel corner detector approach using sliding ellipses, Proc. Int. Conf. Computer Graphics, Imaging and Visualization, (2009), pp. 193-198.

24. R. K. K. Yip, A Hough transform technique for the detection of reflectional symmetry and skew-symmetry, Pattern Recognition Letters, 21(2) (2000), pp. 117-130.

25. R. K. K. Yip, A Hough transform technique for the detection of parallel projected rotational symmetry, Pattern Recognition Letters, 20(10) (1999), pp. 991-1004.

26. R. K. K. Yip, W. C. Y. Lam, P. K. S. Tam and D. N. K. Leung, A Hough transform technique for the detection of rotational symmetry, Pattern Recognition Letters, 15(9) (1994), pp. 919-928. 